\title{
Intensificación de la ganadería porcina y problemas medioambientales en la Conca de Tremp (Pirineo Catalán)
}

\author{
José Borrás Ferrán y José Sánchez Sánchez
}

\begin{abstract}
RESUMEN
La Conca de Tremp, situada en la comarca montañosa catalana del Pallars Jussà, es una de las áreas donde más se ha incrementado la ganadería porcina en régimen estabulado en las últimas tres décadas. Los impactos medioambientales negativos derivados de este sistema de producción ganadera son muy considerables, especialmente los relacionados con el vertido de purines que ha provocado un alto grado de nitrificación de las
\end{abstract} aguas.

Análisis pormenorizados demuestran que el nivel de nitrificación de las aguas en la Conca de Tremp ha alcanzado un nivel muy alto, con las consecuencias que ello tiene en la salud de las personas. Atendiendo a las exigencias del desarrollo sostenible, única forma de garantizar el futuro de las comarcas de montaña, se apuntan soluciones para paliar los graves problemas generados por la intensificación de la ganaderia porcina.

\section{SUMMARY}

The Tremp Basin, placed in the mountainous Catalonian region of the Pallars Jussà, is one of the areas where stabled pig cattle raising has been more increased in the last three decades.

Negative environmental impacts, derived from this cattle raising production system are greatly substantial, specially the ones related to purine wastes which have caused a high degree of nitrification of the waters.

Detailed analyses show that the degree of nitrification of the waters in the Tremp Basin has reached a very high level, with all that this implies for human health. Attending to the requirements of a sustainable development, which is the only way of granting a future for the mountainous regions, this paper hints at some solutions to lessen the serious problems generated by the intensification of pig cattle raising. 
Conca de Tremp, Pallars Jussà, Áreas de montaña, Ganadería porcina, Medio ambiente, Nitrificación de las Aguas, Desarrollo sostenible.
Conca de Tremp, Pallars Jussà. Mountainous regions, Pig cattle raising, Environment, sustainable development.

\section{INTRODUCCIÓN}

En las décadas pasadas el productivismo invadió toda la actividad económica sin tomar en consideración ningún tipo de costos medioambientales. Algunas comarcas catalanas, como otras muchas de España, han intensificado las prácticas agrícolas y ganaderas, con el afán de aumentar las producciones y los rendimientos, como si éstos fuesen los únicos componentes del desarrollo económico.

Hoy se está comprobando que ciertas prácticas productivas en el ámbito rural tienen efectos ecológicos negativos y ponen en peligro el equilibrio medioambiental del entorno. Esto es mucho más grave en las áreas de montaña, como es la comarca del Pallars Jussà, a la que pertenece la Conca de Tremp, área a la que se refiere este trabajo. El nuevo concepto de desarrollo sostenible exige que el crecimiento económico no ponga en peligro ni deteriore el medio ambiente; esto nos lleva al replanteamiento de ciertas prácticas ganaderas, como la ganadería porcina intensiva, cuyos impactos medioambientales, a través sobre todo de la generación de purines, son tan negativos que es necesario encontrar soluciones antes de que sea demasiado tarde.

\section{INTENSIFICACIÓN DE LA GANADERÍA PORCINA EN LA CONCA DE TREMP}

Situada en el Pirineo de la provincia de Lérida y en la comarca del Pallars Jussà, la Conca de Tremp constituye una unidad geográfica de unos $210 \mathrm{Km}^{2}$ en torno a la villa de Tremp que se extiende a ambos lados del río Noguera Pallaresa, en el sector suroccidental pirenaico catalán (mapa de la figura 1).

En general, las comarcas pirenaicas catalanas han conocido un profundo declive de la ganadería tradicional. En algunas ocasiones, la reconversión turística ha sido brutal y una gran parte de los pastos de montaña situados en los pisos alpinos y subalpinos se están degradando por su in- frautilización, causada por el abandono de las actividades ganaderas. 


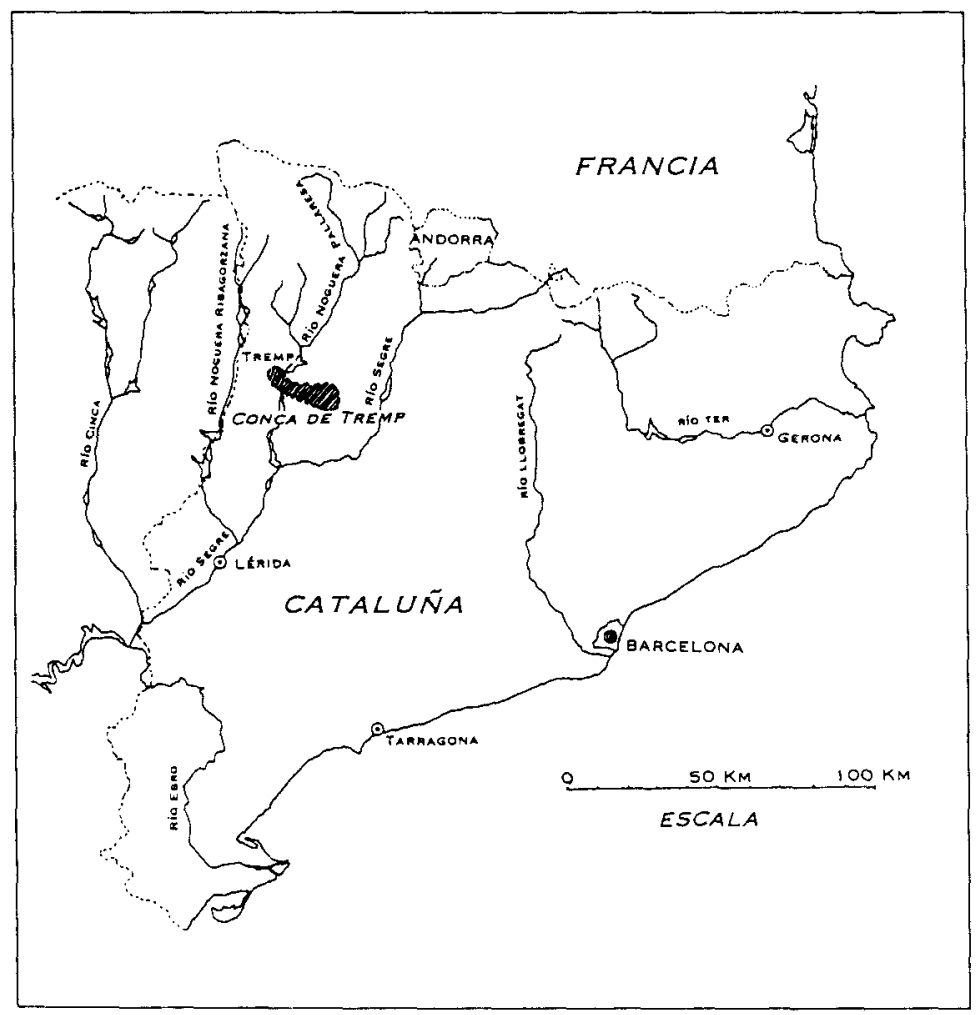

Figura 1. Localización de la Conca de Tremp en Cataluña.

Este proceso es el que ha ocurrido en una comarca situada más al norte, la Val d'Aran, que posee en la actualidad un censo ganadero de menos de 1.800 cabezas de ganado bovino y de menos de 6.000 de ganado ovino, cifras que representan menos de la cuarta parte del censo ganadero existente en la década de los sesenta (ANUARI ESTADÍSTIC DE CATALUNYA, 1997; Solé Sabarís, L., 1964:36).

Esta situación contrasta con la que se presenta en algunas áreas montañosas situadas en lugares de menos atractivo turístico del Prepirineo catalán, en las comarcas del Solsonés, del Bergadà y del Pallars Jussà, y que han conocido un desarrollo extraordinario del subsector porcino estabulado desde los años setenta.

En la actualidad la Conca de Tremp, que ocupa sólo un $15 \%$ de la superficie comarcal, posee una gran importancia agrícola y ganadera, ya que comprende las tres cuartas partes de los campos de cultivo de cereal y al- 
mendro y las dos terceras de la cabaña ganadera total del Pallars Jussà. La Conca de Tremp posee un $79,1 \%$ de los efectivos del censo porcino comarcal y constituye una de las áreas ganaderas catalanas de mayor densidad por habitante y kilómetro cuadrado.

El cuadro $n^{\circ} 1$ y el gráfico de la figura 2 permiten comprobar el gran desarrollo experimentado por el subsector porcino estabulado en la Conca de Tremp en los últimos 35 años.

Cuadro 1. Evolución de los distintos sectores ganaderos en la Conca de Tremp en el período 1960-1995

\begin{tabular}{lrrrr}
\hline & 1960 & 1978 & 1986 & 1995 \\
\hline Censo porcino & 1.400 & 15.630 & 30.600 & 56.120 \\
№ de unidades ganad. porcinas & 350 & 3.750 & 7.330 & 13.450 \\
Censo ovino & 12.000 & 22.000 & 23.000 & 29.900 \\
Número de U.G. ovinas & 1.200 & 2.200 & 2.300 & 2.990 \\
№ de U.G. restantes subsectores & 1.850 & 1.550 & 1.870 & 1.860 \\
№ total de unidades ganaderas & 3.400 & 7.500 & 11.500 & 18.300 \\
Porcentaje del sector porcino & $10 \%$ & $50 \%$ & $64 \%$ & $74 \%$ \\
Unid. ganaderas / habitante (*) & 0,8 & 2,7 & 4,6 & 8,2 \\
\hline
\end{tabular}

(*) Se considera la Conca de Tremp rural, excluyendo la población de Tremp, la capital comarcal. Fuentes: Solé Sabarís, L. 1964; Bernadó Murgó, R. et. al., 1981; Feixa, L., 1991.

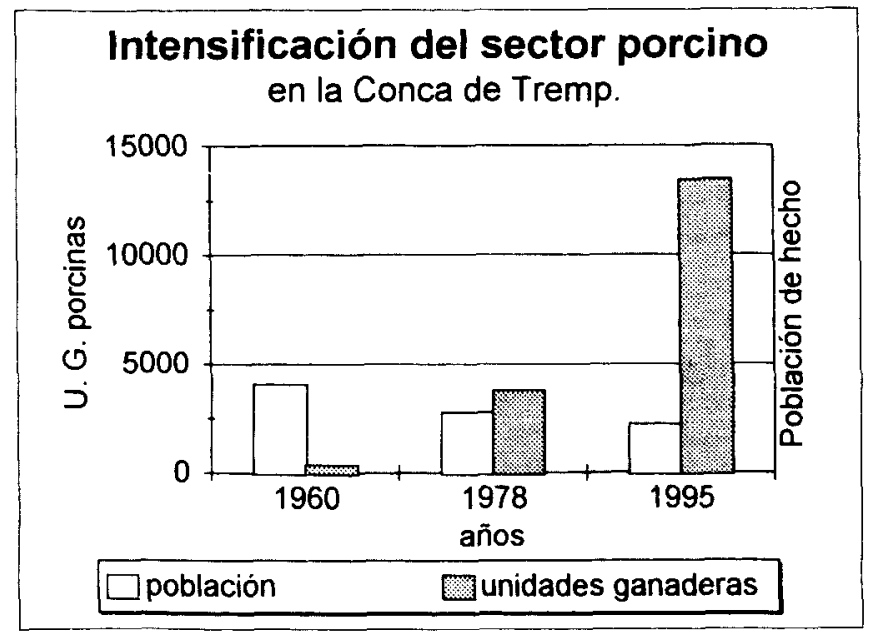

Fuente: Elaboración propia a partir de Nomenclátores de población de Lérida y del Cuadro I.

Figura 2. Desarrollo del sector ganadero porcino en la Conca de Tremp (1960-1995). 


\section{LOS IMPACTOS EN EL MEDIO AMBIENTE PRODUCIDOS POR LAS GRANJAS DE CERDOS ESTABULADOS}

Desde un punto de vista global, resulta posible evaluar el impacto ambiental producido por las granjas de cerdos en explotación intensiva de una determinada área geográfica. Para ello se deben identificar y cuantificar todas las cargas ambientales de los procesos y de los productos que precisa el normal funcionamiento de la granja (fundamentalmente el pienso utilizado en la alimentación porcina) y también la energía necesaria para la cría de cerdos en estas granjas.

Por otra parte, también resulta necesario poder cuantificar los residuos vertidos al exterior de las granjas y estudiar los distintos impactos producidos en el medio ambiente, comparándolos con otros métodos alternativos de crianza en extensivo.

El cuadro $n^{\circ} 2$ refleja una primera aproximación para identificar los impactos ambientales que conlleva la cría de cerdos en régimen intensivo.

Cuadro 2. Inputs y Outputs de la cría de cerdos en régimen intensivo en la Conca de Tremp

Energia y superficie necesaria para la producción del pienso preciso (45.000 tons. de cebada/año) $\Rightarrow$

Materiales y energía empleados en construcción de las granjas $\Rightarrow$ Superficie de suelo ocupada por las granjas y vertidos de purin $\Rightarrow$

Energía precisa para transportar las materias primas y los cerdos $\Rightarrow$

Agua, productos veterinarios $\Rightarrow$

GRANJAS DE CRIA
DE CERDOS EN
RÉGIMEN INTENSIVO
EN LA CONCA
DE TREMP

$\Rightarrow 0,14 \mathrm{Hm}^{3}$ purín/año.

$\Rightarrow$ impacto visual, auditivo y olfativo de las granjas y de los purines vertidos.

$\Rightarrow$ impacto en el resto de los sectores económicos.

$\Rightarrow$ impacto en la salud de las personas de la Conca.

$\Rightarrow$ contaminación de los acuíferos y del suelo.

Del cuadro precedente cabe destacar:

1) Los altos consumos de energía para la obtención y el transporte de las materias primas necesarias para la fabricación de los piensos (principalmente en forma de derivados del petróleo), al estar los cerdos alimentados por cereales producidos en Norteamérica, como la soja, y por harinas de pescado procedentes del Pacífico. Por otra parte, las calorías contenidas en el pienso suministrado como alimento para el ganado, estimadas en unas $1.880 \mathrm{cal} / \mathrm{Kg}$ de cebada (unidad forrajera de referencia), permitirían cubrir las necesidades energéticas de una población de 80.000 habitantes, si se destinara la tierra a producir cereales para consumo humano. 
2) El gran impacto visual, auditivo, olfativo y medioambiental de las granjas, al estar a menudo situadas muy cerca del casco antiguo de los pueblos. Los desasosegadores chillidos de los cerdos cuando reciben sus raciones diarias de alimento y los malos olores de las granjas constituyen un freno para un posible futuro desarrollo turístico de la Conca de Tremp. De todos modos, el impacto más importante en el medio ambiente viene causado por los vertidos de sus purines, tal como queda reflejado en la sección siguiente.

3) Los mayores costos del transporte, al estar situadas las explotaciones ganaderas porcinas en localizaciones relativamente alejadas de las grandes fábricas de pienso y de los grandes mataderos industriales.

\section{LOS IMPACTOS EN EL MEDIO AMBIENTE PRODUCIDOS POR EL VERTIDO DE LOS PURINES: LA NITRIFICACIÓN DE LAS AGUAS}

En los países occidentales, el vertido y posterior filtración de los excrementos ganaderos, utilizados en muchas ocasiones como fertilizantes agrícolas por su alto contenido en nitrógeno, fósforo y potasio, originan la mayor parte de los problemas de deterioro de la calidad de los suelos agrícolas y de las aguas superficiales y subterráneas.

Para estudiar el impacto producido por el subsector porcino resulta necesario conocer tanto la composición del censo ganadero porcino total de la Conca como la gestión de los purines producidos en las granjas.

A diferencia de lo que acontece en otras áreas de Europa como en Holanda, en donde los residuos ganaderos suponen el $100 \%$ del aporte del abono nitrogenado de los campos de maíz (Den Ouden, G. y Tirion, H.B., 1993), en la Conca de Tremp es muy escasa la complementariedad del sector agrícola y ganadero y con frecuencia los purines porcinos son vistos más como residuos inútiles que conviene eliminar de la forma que sea, que como valiosos fertilizantes.

Consecuentemente, la mayor parte de los purines de las granjas de la Conca son vertidos directamente al cauce de los ríos o en algunos campos de almendros o campos en barbecho muy localizados que reciben dosis masivas de purín porcino, sobrepasando con frecuencia los 100 metros cúbicos de purín/ $\mathrm{Ha}$, lo que provoca la aparición de costras oscuras de purín de unos $2 / 3 \mathrm{~cm}$ de espesor, de forma similar a lo que ocurre en otros lugares de alta densidad porcina de la geografía española, como en la provincia de Segovia (Rodríguez García, A., 1994; Flor Masedo M. y Lobete Huertas, F., 1996). Estas aplicaciones masivas de purín incrementan los niveles de los fosfatos y de los nitratos de las aguas superficiales y subterráneas. 
Un efecto muy importante en el medio ambiente del vertido de los purines en los ríos es el de la eutrofización, proceso en el que el exceso de nutrientes (principalmente nitratos y fosfatos) produce un excesivo crecimiento de algas y plantas acuáticas, cuya descomposición provoca la desoxigenación del río y una pérdida de la biodiversidad de especies presentes en el agua, que se vuelve maloliente y desprovista de vida. En todo el territorio de los Países Bajos, y con la finalidad de limitar sus efectos nocivos en el medio ambiente, está previsto disminuir los vertidos de purín para el año 2004 a valores inferiores a los $170 \mathrm{Kg}$ de N./Ha/año (Den Ouden, G. y Tirion, H.B., 1993), unas cifras que se triplican con facilidad en numerosas localidades de la geografía española.

Según el censo ganadero de 1995, la Conca de Tremp contaba con un total de 56.120 cerdos, de los cuales una cuarta parte eran adultos 0 reproductores y las tres cuartas partes restantes eran cerdos de engorde de distintas edades (Comunicación personal de la oficina comarcal del DARP, en Tremp, y ANUARI ESTADÍSTIC DE LA GENERALITAT DE CATALUNYA, 1997).

A partir de este censo y de la consulta de manuales especializados (Concellón Martínez, A., 1977, Poulenc, J.G., 1982 o Prats, I., 1995), ha sido posible confeccionar el cuadro $n^{\circ} 3$, que complementa los datos del cuadro $n^{\circ} 1$. Conociendo la composición del purín porcino de la Conca de Tremp, resulta posible cuantificar el impacto ambiental producido por cerdo y día y por la totalidad de la cabaña porcina de la Conca de Tremp.

Cuadro 3. Características del Purín producido por tipo cerdo en la Conca de Tremp, diariamente

\begin{tabular}{lcccc}
\hline & $\begin{array}{l}\text { CERDO } \\
\text { REPRODUCTOR }\end{array}$ & $\begin{array}{c}\text { CERDO DE 30 } \\
\text { KGS. (3 meses) }\end{array}$ & $\begin{array}{c}\text { CERDO DE 95 } \\
\text { KGS. (6 meses) }\end{array}$ & $\begin{array}{c}\text { CERDO MEDIO } \\
\text { CONCA DE T. }\end{array}$ \\
\hline Producción purín & $12,5 \mathrm{I}$ & $3 \mathrm{l}$ & $8,5 \mathrm{I}$ & $6,9 \mathrm{I}$ \\
N. total excretado & $65 \mathrm{~g}$ & $23 \mathrm{~g}$ & $70 \mathrm{~g}$ & $59 \mathrm{~g}$ \\
P2O5 total excretado & $50 \mathrm{~g}$ & $18 \mathrm{~g}$ & $51 \mathrm{~g}$ & $35 \mathrm{~g}$ \\
$\mathrm{~K}_{2} \mathrm{O}$ & $29 \mathrm{~g}$ & $7 \mathrm{~g}$ & $20 \mathrm{~g}$ & $17,5 \mathrm{~g}$ \\
Cobre $(\mathrm{Cu})$ & $0,1 \mathrm{~g}$ & $0,2 \mathrm{~g}$ & $0,6 \mathrm{~g}$ & $0,4 \mathrm{~g}$ \\
Zinc $(\mathrm{Zn})$ & $0,8 \mathrm{~g}$ & $0,2 \mathrm{~g}$ & $0,6 \mathrm{~g}$ & $0,5 \mathrm{~g}$ \\
\hline
\end{tabular}

Fuente: Elaboración propia a partir de Prats, I., 1995 y Poulenc, J.G., 1982.

Si nos ceñimos al impacto producido por el subsector porcino en el espacio geográfico de la Conca de Tremp, tenemos una producción diaria de unos 388.000 litros de purín, lo que representa más de 140.000 metros cú- 
bicos al año, con un vertido anual de unas 1.000 toneladas N./año, que equivalen a una media de unos $47 \mathrm{Kg}$ de $\mathrm{N} . / \mathrm{Ha}$. para el conjunto de la Conca, lo que supone una cifra bastante elevada al tratarse de una área de montaña con un medio ambiente valioso y sensible.

El cerdo que se ceba sólo asimila una tercera parte del nitrógeno total que ingiere. Las dos terceras partes son excretadas: una quinta parte por vía fecal y el resto por la vía urinaria. Asimismo, el cerdo sólo asimila un $30 \%$ del fósforo suministrado en los alimentos y 10 mismo ocurre con el cobre y el zinc añadidos en el pienso como factores de crecimiento y que son sólo asimilados en cantidades minimas.

En los purines de cerdo algo más de un $60 \%$ del nitrógeno excretado está en forma amoniacal, estando el resto combinado en la materia orgánica. De este modo, los purines de cerdo constituyen una de las principales fuentes de emisión atmosférica de amoníaco. Así, en todo el territorio de Holanda, en el período de un año, la producción de estiércol fue de 86 millones de toneladas, estimándose en 200.000 las toneladas de amoníaco emitidas (Den Ouden, G. y Tirion, B., 1993).

Si bien una pequeña fracción del nitrógeno de los purines se libera a la atmósfera en forma de gas nitrógeno molecular $\left(\mathrm{N}_{2}\right)$, la mayor parte de este elemento se oxida a nitritos y posteriormente a nitratos por acción de las bacterias nitrificantes existentes en el suelo. Este es el origen del problema de la nitrificación o creciente contaminación por los nitratos de las aguas superficiales y subterráneas, tanto en la Conca de Tremp como en otras zonas ganaderas y también en algunas áreas rurales españolas de horticultura intensiva.

En los últimos años han sido muchas en nuestro país las voces de alerta por la nitrificación creciente tanto de las aguas superficiales como de las subterráneas y las opiniones expresadas por algunos especialistas y divulgadores han conocido una creciente difusión (SERVICIO GEOLÓGICO, M. ${ }^{\circ}$ DE O.P., 1991; Miracle, Mª R., 1993; Gallart, I., 1997; Custodio, E., 1997).

La nitrificación en la Conca de Tremp viene sobre todo causada por los vertidos de purines porcinos (unos $140.000 \mathrm{~m}^{3} / \mathrm{año}$ ) en lugares muy localizados situados en las cercanías de las granjas. Asimismo, el estiércol producido por otros tipos de ganado, los vertidos de las aguas residuales de las poblaciones más importantes como Tremp o Isona y la percolación o arrastre de los abonos nitrogenados en los campos de cultivo contribuyen igualmente a incrementar la nitrificación, aunque su importancia relativa es mucho menor que la de los purines de cerdo. 
Intensificación de la ganaderia porcina y problemas medioambientales en...

La medición de los niveles de nitratos de las aguas superficiales y subterráneas de la Conca de Tremp ha sido una de las tareas realizadas por Borrás Ferrán, J. en sus estudios sobre las posibilidades de aplicar propuestas de desarrollo socioeconómico sostenible a medio y largo plazo en la comarca de montaña del Pallars Jussà, en el Pirineo catalán. El cuadro $n^{\circ} 4$ muestra los resultados de las mediciones efectuadas en los años 1996 y 1997, pudiéndose comprobar cómo los niveles de nitrato aumentan conforme pasa el tiempo.

Cuadro 4. Nitrificación creciente de los acuíferos de la Conca de Tremps (Enero 1996/Abril 1997)

\begin{tabular}{lcccccc}
\hline & $\begin{array}{l}\text { NITRATO } \\
\text { INICIO } \\
\text { (MG/L) }\end{array}$ & $\begin{array}{l}\text { NITRATOS } \\
\text { FINAL } \\
\text { (MG/L) }\end{array}$ & $\begin{array}{l}\text { NIVEL MÁX. } \\
\text { NITRATOS* } \\
\text { (MG/L) }\end{array}$ & $\begin{array}{l}\text { NIVEL NO } \\
\text { MEDIO } \\
\text { (MG/L) }\end{array}$ & $\begin{array}{l}\text { ALTITUD } \\
\text { EN } \\
\text { METROS }\end{array}$ & $\begin{array}{l}\text { NÚMERO } \\
\text { DE }\end{array}$ \\
MEDICIONES
\end{tabular}

Fuentes: mediciones efectuadas en la Conca de Tremp por José Borrás Ferrán. Las tres últimas filas de la tabla se corresponden con los datos obtenidos a partir del ANUARI ESTADÍSTIC DE LA GENERALITAT DE CATALUNYA, 1996.

(1): valor mínimo. (*) Esta fuente está situada en el centro de Tremp, en la parte septentrional de la Plaza Soldevila.

A partir de los datos contenidos en el cuadro no 4 y del mapa de la figura 3 , en donde se señalan los puntos de muestreo, podemos extraer las siguientes conclusiones: 


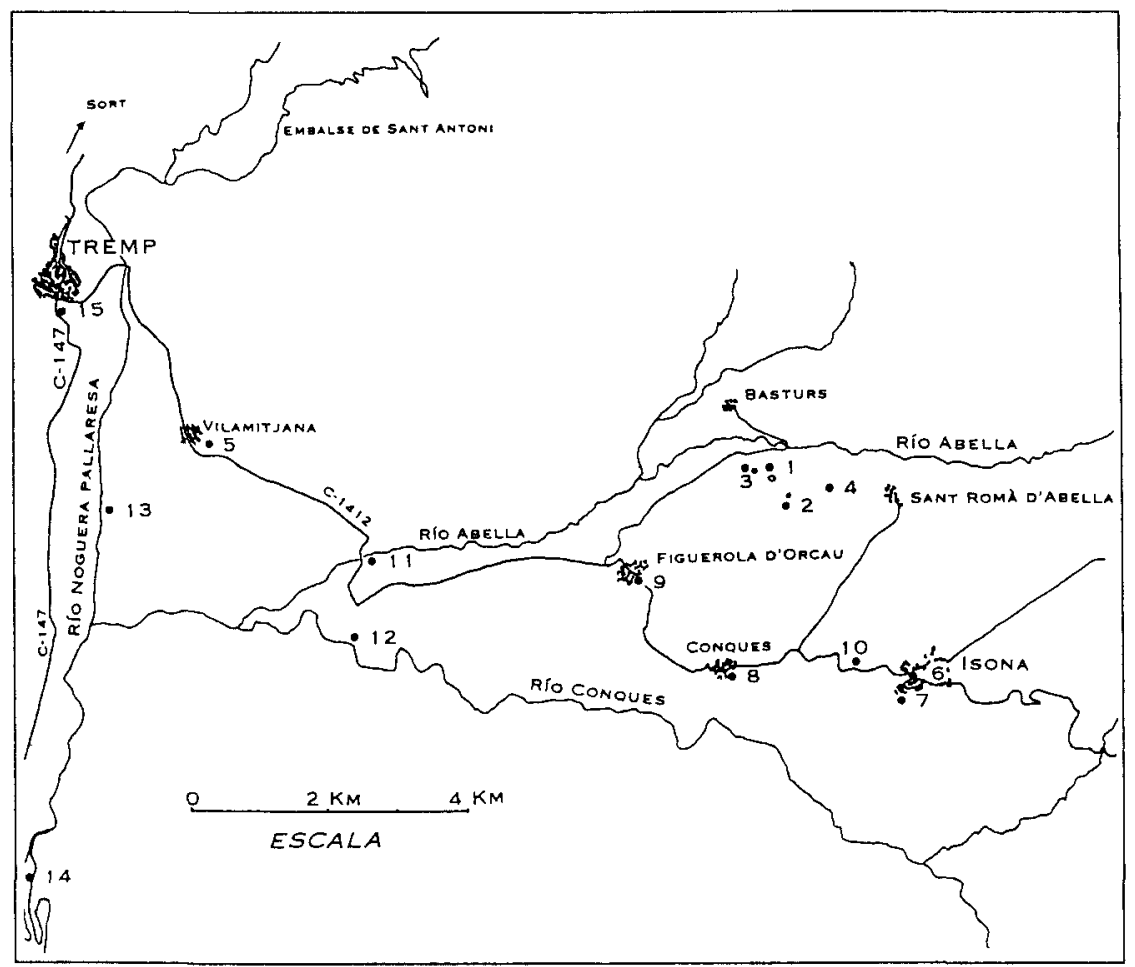

Figura 3. Localización de los puntos de muestro en el sector oriental de la Conca de Tremp.

1) Los ríos más contaminados son los que atraviesan las áreas con más densidad de granjas de ganado porcino estabulado. En la Conca de Tremp, los ríos Conques y Abella, afluentes por la izquierda del río Noguera Pallaresa, poseen cerca de sus respectivas desembocaduras un contenido en nitratos en torno a los $80 \mathrm{mg} / \mathrm{litro}$.

2) De todos modos, las mayores concentraciones de nitratos se dan en las aguas subterráneas y así algunas fuentes de los pueblos de la Conca de Tremp y del mismo Tremp poseen un contenido en torno a los $180 / 250 \mathrm{mg} /$ litro de nitratos. Estas cifras son algo inferiores a los máximos detectados en acuíferos subterráneos en las comarcas de Osona y del Maresme, situadas al norte y al nordeste de Barcelona (unos 500-800 mg/litro en el año 1996), pero son similares a los valores máximos detectados en la península en 1990 (SERVICIO GEOLÓGICO, MํDE O.P., 1991; Miracle, M.̊ R., 1993; Gallart, I., 1997).

3) En la Conca de Tremp los niveles naturales de nitratos de las aguas superficiales y subterráneas, sin ningún tipo de acción antrópica, no suelen so- 
brepasar los 5-10 mg/litro. En la actualidad tan sólo los acuíferos de la Conca de Tremp situados a una gran profundidad, como los que alimentan a los emisarios de las lagunas kársticas de Basturs o los que alimentan los acuíferos de la red de Isona o de Vilamitjana poseen estos valores más bajos.

\section{EL EFECTO DE LOS NITRATOS EN LA SALUD DE LAS PERSONAS}

Los nitratos, por sí mismos, no resultan tóxicos para los mamíferos o las personas. El problema estriba en que una vez ingeridos pueden reducirse a nitritos. Ya en la sangre, los nitritos provocan la oxidación del $\mathrm{Fe}^{++}$a Fe $\mathrm{Fe}^{+++}$ de la molécula de hemoglobina, que pierde así su capacidad para transportar el oxígeno, provocando una asfixia conocida como metahemoglobinemia. Los bebés de menos de seis meses son especialmente sensibles a la metahemoglobinemia.Se han descrito casos de muertes de recién nacidos alimentados con leche en polvo poco ácida, utilizando aguas con un contenido en nitratos superior a los $60 \mathrm{mg} / \mathrm{l}$ (OCDE, 1986).

Por otra parte, los nitritos pueden reaccionar en la sangre con las aminas provenientes del metabolismo de las proteínas y formar unas sustancias presumiblemente cancerígenas: las nitrosaminas, aunque todavía se discute si existe o no una relación clara y directa entre la ingestión total de nitratos, la sintesis de nitrosaminas y el riesgo de desarrollar tumores malignos (Higgins, I., 1975; García Badell, J.J., 1987; SHANXI CÁNCER INSTITUTE, 1995).

El contenido máximo de nitratos en las aguas potables debería ser inferior a los $50 \mathrm{mg} /$ litro o incluso a los $25 \mathrm{mg} /$ litro, si se desean evitar los perniciosos efectos de las aguas con niveles altos de nitratos para la salud humana.

De esta forma, todas las legislaciones (tanto la vigente en nuestro país como la de los otros estados), establecen de forma clara los criterios de potabilidad de las aguas, limitando la concentración en las aguas potables de nitratos y otros contaminantes. El cuadro 5, los R.D. 1423/82 y $1138 / 90$, las Directivas comunitarias 75/440/CEE y $80 / 778 /$ CEE y algunos libros especializados como Higgins, I., 1975 y O.C.D.E., 1986, contienen los criterios de la calidad que deben reunir las aguas potables.

Finalmente, conviene señalar tres elementos adicionales de interés a tener en cuenta en el estudio del impacto de la nitrificación en la salud de las personas:

1) En primer lugar, el incremento exponencial de la contaminación de algunos acuíferos subterráneos, lo que posibilita que en algunos casos, en un periodo de tiempo de tan sólo unos 12 meses se duplique, triplique o cuadri- 
plique su contenido en nitratos. Este hecho se debe a que la contaminación de los acuíferos subterráneos se produce de un modo bastante súbito, cuando el frente de nitratos que ha ido percolando desde la superficie alcanza el acuífero subterráneo, normalmente unos años más tarde de ser vertidos. Este es el hecho que ha sucedido en la fuente de Conques, cuyo nivel de nitratos ha subido desde los $70 \mathrm{mg} / \mathrm{l}(3 / 1996)$ hasta los $280 \mathrm{mg} / /$ (4/1997).

2) En segundo lugar, tal como atestigua el cuadro 4, una misma comarca presenta valores muy dispares en el contenido de nitratos de sus aguas superficiales y subterráneas. Estos valores tan dispares se deben tanto a factores de geografía humana (localización de los vertidos en áreas muy concretas) como a las diferencias de geografía física, ya que la velocidad de lavado, de unos pocos metros al año, es muy variable según la composición, textura y estratigrafía del terreno en donde se vierten los purines, y según el régimen pluviométrico.

3) En tercer lugar, es preciso considerar la exportación de la nitrificación superficial y subterránea hacia las comarcas y ciudades más pobladas y situadas a menor altitud, como son Lérida capital o Balaguer.

\section{OTROS IMPACTOS MEDIOAMBIENTALES DE LOS PURINES PORCINAS EN LA CONCA DE TREMP}

Los vertidos de purines no sólo tienen efectos en el incremento de la nitrificación y de los niveles de nitritos y nitratos en las aguas superficiales y subterráneas de la Conca de Tremp.

En primer lugar, cabe considerar además el incremento de los niveles de fosfatos en el suelo y en las aguas en donde va a parar el purín porcino, cuyo contenido en P2O5 es de unos 5 gr/litro (Prat, l., 1995 y cuadro 3 ). Las aguas contaminadas con fosfatos poseen niveles de P2O5 superiores a los 0,2 mg/litro y provocan la eutrofización de las aguas en una acción sinérgica y conjunta con los nitratos también presentes.

Precisamente, con el fin de evitar la eutrofización de las aguas y una acidificación excesiva de los suelos, la legislación holandesa limita el vertido de purines porcinos a menos de $125 \mathrm{Kg}$ de P2O5/Ha/año. De este modo, se pretende reducir el contenido de fósforo a valores inferiores a los $0,3 \mathrm{mg} / \mathrm{litro}$ de P2O5 en aguas superficiales y a los $0,9 \mathrm{mg} / \mathrm{litro}$ de P2O5 en aguas subterráneas. En Cataluña, los ríos que atraviesan las áreas de un desarrollo agrícola y ganadero más intensivo son los que poseen concentraciones de P2O5 superiores a $1 \mathrm{mg} /$ /itro, como ocurre en Torres de Segre al final del curso del río Segre (ANUARI ESTADÍSTIC DE CATALUNYA, 1997:74). 
Cabe igualmente citar la Directiva comunitaria de $15 / 7$ de 1980 , que establece un nivel guía de 0,4 mg/litro de P2O5 para todas las aguas destinadas al consumo humano y que fija una concentración máxima admisible de $5 \mathrm{mg} /$ /itro de P2O5. Este es un valor que seguramente superan en la actualidad numerosos acuíferos subterráneos de la Conca de Tremp y el mismo río Conques en su curso más bajo (mapa de la figura 2).

En segundo lugar, cabe señalar la elevada contaminación de metales pesados como el cobre, el manganeso o el zinc, presente en muchas áreas rurales de ganadería porcina intensiva tanto en sus suelos como en sus aguas superficiales y subterráneas y que está causada por los vertidos de los purines porcinos.

Tanto el cobre como el zinc y el manganeso se encuentran en los purines de cerdo de las explotaciones de engorde en concentraciones comprendidas entre los $45 \mathrm{mg} / \mathrm{l}$ y los $75 \mathrm{mg} / \mathrm{l}$. Resulta por tanto previsible considerar un incremento de la contaminación de estos metales en las aguas y en los suelos de la comarca y relacionarlo con los efectos sinérgicos negativos de los nitratos a nivel ganadero y sanitario (Ayers, R.S. y Weccot, D.W., 1976; SHANXI CANCER INSTITUTE, 1995).

Cuadro 5. Calidad de las aguas según su uso

\begin{tabular}{|c|c|c|c|c|c|}
\hline & $\mathrm{PH}$ & NITRATOS & MANGANESO & ZINC & COBRE \\
\hline Purín granja cebado (1) & 8 & $34 \mathrm{~g} / /\left(^{*}\right)$ & $46 \mathrm{mg} / 1$ & $71 \mathrm{mg} / \mathrm{l}$ & $67 \mathrm{mg} / \mathrm{l}$ \\
\hline Agua de riego (2) & $6,5 \cdot 8,4$ & $30 \mathrm{mg} / \mathrm{l}$ & $0,2 \mathrm{mg} / \mathrm{l}$ & $2 \mathrm{mg} / \mathrm{l}$ & $0,2 \mathrm{mg} / \mathrm{l}$ \\
\hline Agua para el ganado (3) & $6-9$ & $100 \mathrm{mg} / \mathrm{l}$ & $0,1 \mathrm{mg} / \mathrm{l}$ & $20 \mathrm{mg} / \mathrm{l}$ & $0,5 \mathrm{mg} / \mathrm{l}$ \\
\hline Agua prepotable A1 (4) & $6,5 \cdot 8,5$ & $25 \mathrm{mg} / 1$ & $0,05 \mathrm{mg} / \mathrm{l}$ & $0,5 \mathrm{mg} / \mathrm{l}$ & $0,02 \mathrm{mg} / \mathrm{l}$ \\
\hline Agua prepotable A3 (4) & $5,5-9$ & $50 \mathrm{mg} / \mathrm{l}$ & $1 \mathrm{mg} / \mathrm{l}$ & $1 \mathrm{mg} / \mathrm{l}$ & $1 \mathrm{mg} / \mathrm{l}$ \\
\hline Agua potable, mg/l (4) & $6,5 \cdot 9,5$ & $25 / 50$ & $0,02 / 0,05$ & $0,1 / 5$ & $0,1 / 1,5$ \\
\hline Agua potable (USA) (5) & - & $45 \mathrm{mg} / \mathrm{l}$ & $0,05 \mathrm{mg} / \mathrm{l}$ & $5 \mathrm{mg} / \mathrm{l}$ & $1 \mathrm{mg} / \mathrm{l}$ \\
\hline Contenido corporal total & - & - & $12 \mathrm{mg}$ & $2.300 \mathrm{mg}$ & $72 \mathrm{mg}$ \\
\hline Vida media (5) & - & - & 17 dias & 933 dias & 80 dias \\
\hline Agua red Isona & 7,4 & 15 & ? & $?$ & $?$ \\
\hline Agua río Conques & $8,0 / 8,2$ & $90 / 100$ & $?$ & $?$ & $?$ \\
\hline Aquíteros de la Conca & $7,2 / 7,6$ & $15 / 300$ & $?$ & $?$ & $?$ \\
\hline Agua río N. Pallaresa (6) & $7,5 / 7,7$ & $10 / 20$ & $?$ & $?$ & $?$ \\
\hline Aguas en USA (mg/) (5) & - & $0,1 / 500$ & $0,0003 / 3$ & $0,002 / 1,2$ & $0,001 / 0,28$ \\
\hline Aguas en USA valores medios & - & 20 & 0,058 & 0,064 & 0,015 \\
\hline
\end{tabular}

$\left(^{\star}\right)$ : Cantidad equivalente al contenido en nitratos del nitrógeno total del purín.

Fuentes: (1): Se trata de un valor medio obtenido en 1991, a partir de análisis en instalaciones ganaderas de cebado de cerdos (Prats, I., 1995); (2), (3): Ayers, R.S. y Weccot, D.W., 1976; (4): 80 / 778 / CEE y R.D 1.138/1990; (5): Stoker, H. S., 1981; (6): ANUARI ESTADÍSTIC DE CATALUNYA, 1997. 
El cuadro $n .-5$ resume los valores máximos admisibles de algunos parámetros de las aguas en distintos ámbitos geográficos y usos diferentes. En él se evidencia tanto el desconocimiento actual de los niveles de metales pesados en la Conca de Tremp como los muy altos niveles de nitratos de sus aguas, excesivos a menudo incluso para su uso ganadero. En nuestro país son todavía poco frecuentes las investigaciones en el medio rural con mediciones de microcontaminantes como antibióticos para uso ganadero o metales pesados contenidos en los suelos y en las aguas superficiales y subterráneas.

De todos modos, conviene destacar el estudio realizado en Segovia sobre la contaminación por cobre y zinc de suelos por purines de cerdo (Masedo, M.F. y Lobete Huertas, F., 1996) y también la normativa europea, que limita la presencia de zinc, cobre y manganeso en el pienso porcino a $250 \mathrm{ppm}(250 \mathrm{mg} / \mathrm{Kg})$. En los países con una mayor tradición ganadera intensiva y más contaminados como Holanda, los fabricantes locales de pienso rebajan todavía más el límite fijado en esta normativa y lo sitúan a 80 ppm de zinc (Den Ouden, G. y Tirion, H.B., 1993).

Finalmente, hay que señalar también que a largo plazo la acumulación de cobre en los suelos puede resultar tóxica en algunos tipos de plantas, pero sobre todo para el ganado ovino que paste en prados, cuyos vegetales contengan cobre acumulado (OCDE, 1986).

\section{CONCLUSIONES}

La intensificación de la ganadería porcina en la Conca de Tremp en las últimas décadas ha provocado un proceso de contaminación creciente que es necesario detener.

La mejor forma de deshacerse de los purines porcinos, y en general de los residuos ganaderos en cualquier espacio geográfico, es emplearlos como fertilizantes en dosis adecuadas, a pesar de los inconvenientes que presentan, como su olor desagradable y la presencia de metales contaminantes y tóxicos en elevadas concentraciones como el cobre, el manganeso o el zinc.

El alto contenido de nitratos hace que las aguas de los ríos Conques y Abella sean inadecuadas para el consumo humano y provoquen al mismo tiempo una importante eutrofización en sus aguas y en las del río Noguera Pallaresa. Pero, éstas podrían ser reutilizadas para el riego de cultivos que precisen elevados aportes de nitrógeno como el maíz. De acuerdo con los cálculos de Prats, I. para la provincia de 
Lérida (1995) y de Broadbent y Carlton para California (Ramos Mompo, C., 1993: 11) en las comarcas situadas más al sur (como Segrià o La Noguera) los aportes de nitratos de estas aguas utilizadas en el riego bastarían para cubrir un $60 \%$ de las necesidades totales de fertilizante nitrogenado.

De todos modos, tanto por motivos de sanidad animal como por la escasez de tierras aptas para la agricultura en las áreas de montaña, debería evitarse un mayor incremento de la cabaña porcina en las granjas en toda la Conca de Tremp y en especial en su periferia más montañosa y de mayor valor ecológico. Además, la Administración pública debería poner mayor empeño y competencia para impedir los vertidos directos de purines en los cauces de los ríos y torrentes.

En la actualidad, y sobretodo a partir del inicio de 1997, una fracción cada vez más importante de los purines producidos en las granjas de la Conca de Tremp es evacuada fuera de la comarca por medio de camiones cisterna que realizan recorridos de más de $100 \mathrm{Km}$, ida y vuelta, con 10 que el impacto de los vertidos se traslada fuera de la comarca del Pallars Jussà. Pero esta exportación de contaminación no es la solución a este problema, ya que agrava la nitrificación de las aguas subterráneas de las tierras bajas de Lérida: muchas empresas ganaderas de Holanda contratan el cebado de sus cerdos a otras empresas situadas al sur de la provincia de Lérida, ahorrándose así los altos costes de la colocación de los purines (Den Ouden, G. y Tirion, H.B., 1993).

Por otra parte, el desarrollo de la ganadería porcina estabulada en las comarcas de montaña, apartadas de los mataderos y de los lugares de consumo y mal comunicadas con las fábricas de pienso, posee unos mayores costos comparativos de transporte; por ello está basada en la máxima productividad económica de los otros factores de producción. A corto plazo, esto fomenta una mentalidad de primar la cantidad en detrimento de la calidad, lo contrario de lo que exige el desarrollo sostenible, indispensable para el futuro de las comarcas de montaña.

Contrariamente a lo que sucede en otras áreas montañosas europeas, en las que se fomentan los productos basados en la agricultura biológica y en la ganadería extensiva, con frecuencia comercializados en la misma área, la Conca de Tremp y la comarca del Pallars Jussà carecen de productos transformados porcinos de calidad, a pesar de ser éste el subsector ganadero más importante.

Una promoción inteligente y responsable de una ganadería extensiva de calidad sería, a la larga, mucho más beneficiosa desde un punto de vista económico y social para toda la comarca. 


\section{BIBLIOGRAFÍA}

ANUARI ESTADÍSTIC DE CATALUNYA, 1997. Ed. Institut d'Estadística de Catalunya, Barcelona.

AYERS, R.S. y WECCOT, D.W. (1976): La Qualité de l'eau en agriculture. FAO paper núm. 29, Ed. FAO, Roma.

BernaRdo Murgo, J. (1981): El Pallars Jussà. Estructura sòcio-econòmica i territorial del Pallars Jussà i de l'Alta Ribagorça. Ed. Caixa de Catalunya, Barcelona.

Concellón Martinez, A. (1977): Ganadería práctica. Ed. R. Sopena, Barcelona.

Custodı, E. (1997): “La contaminación subterránea en España. El agua y el medio ambiente». La Vanguardia Ciencia y salud, 22/2/97. 4-5. Barcelona.

Den OUden, G. y TiRion, H.B. (1993): “La gestión del estiércol en los Países Bajos». Medi Ambient, Tecnologia i Cultura, núm. 7. 22-29. Ed. Departament de Medi Ambient de la Generalitat de Catalunya, Barcelona.

FEIXA, L. (1991): Pla Comarcal de Muntanya 1991-1995: El Pallars Jussà. Ed. Departament de Política Territorial i O.P. Generalitat de Catalunya, Barcelona.

GALLART, I. (1997): "Los purines contaminan la tierra, el agua y el aire". Revista Integral, núm. 210, págs. 14-15, Barcelona.

GARCIA BADELL, J.J. (1987): La contaminación y el equilibrio ecológico. Ed. I.N.I.A., Madrid.

HigGins, I.J. (1975): The chemistry and microbiology of pollution. Ed. Academic Press, London.

MASEDO, M.F. y LoBeTE HUERTAS, F. (1996): Contaminación por metales pesados (Cu y $Z n$ ) en suelos afectados por purines de cerdo en la provincia de Segovia. Biodisponibilidad de estos contaminantes y problemática ambiental. Ed. Caja de Segovia, Segovia.

MiRACLE, M. ${ }^{a}$ R. (1993); “El agua: ¿explotación o conservación?» Medi Ambient, Tecnologia i Cultura, núm. 7. 62-64. Ed. Departament de Medi Ambient de la Generalitat de Catalunya, Barcelona.

O.C.D.E. (1986): Water pollution by fertilizers and pesticides. Ed. OECD Publications, Paris.

POULENC, J.G. (1982): Mémento de l'éleveur de porc. Ed. I.T.P., Paris.

Prats, I. (1995): Manual de gestió dels purins i de la seva reutilització agrícola. Ed. Generalitat de Catalunya, Barcelona.

Ramos Mompo, C., (1993): La agricultura y la contaminación de las aguas por nitrato. Ed. Me. de Agricultura, Pesca y Alimentación, Madrid.

Rodriguez Garcia, A., coord. (1994): Comunidades agrarias como receptoras de un impacto ambiental: el vertido de lisier. Ed. Diputación Provincial de Segovia.

SERVICIO GEOLOGICO del M. ${ }^{\circ}$ de O.P. (1991): "Situación de la contaminación por nitratos en las aguas subterráneas del territorio peninsular y balear". Informaciones y Estudios, núm. 53 , Madrid.

SHANXI CANCER INSTITUTE (1995): A study on the relationship between malgnant tumour mortality and environmental pollution in Beicun countryside of Datong city. Internet.medline, núm. 95300197.

Solé Sabaris, L. (1964): Geografía de Catalunya. Vol. 2. Ed. Aedós; Barcelona.

StOKER, H.S. (1981): Química ambiental. Contaminación del aire y del agua. Ed. Blume, Barcelona. 